\title{
Demographic, Practice and Clinical Management Characteristics of Osteopaths Referring to, and Receiving Referrals from, Podiatrists: Secondary Analysis of a Nationally Representative Sample of Australian Osteopaths.
}

Michael Fleischmann ( $\nabla$ michael.fleischmann@vu.edu.au )

Victoria University

\section{Brett Vaughan}

University of Melbourne

Adam Bird

La Trobe University

Sandra Grace

Southern Cross University

Kylie Fitzgerald

RMIT University

Gopi McLeod

Southern Cross University

\section{Research Article}

Keywords: Interprofessional care, healthcare , podiatrists , osteopaths

Posted Date: January 13th, 2021

DOI: https://doi.org/10.21203/rs.3.rs-133944/v1

License: (c) (i) This work is licensed under a Creative Commons Attribution 4.0 International License.

Read Full License 


\section{Abstract}

\section{Background}

Interprofessional care is paramount in contemporary healthcare practice. How different professions interact, and the characteristics of those practitioners who practice in an interprofessional way are rarely described in the literature. The aim of the current work was to identify the demographic, practice and clinical management characteristics of Australian osteopaths who report referring to, and receiving referrals from, podiatrists

\section{Methods}

The study was a secondary analysis of data from the Osteopathy Research and Innovation Network (ORION). Inferential statistics were generated to identify statistically significant demographic, practice and clinical management characteristics associated with referrals. Significant characteristics were then entered into a backward logistic regression model.

\section{Results}

Nine-hundred and ninety-two Australian osteopaths responded to the questionnaire. Sending referrals to a podiatrist was reported by 651 participants $(65.6 \%)$ and receiving referrals from a podiatrist by 471 participants (47.5\%). Australian osteopaths who reported referring to podiatrists were more likely to report receiving referrals from podiatrists $\left(\mathrm{OR}_{\mathrm{adj}} 10.82\right)$ and use diagnostic imaging $\left(\mathrm{OR}_{\mathrm{adj}}{ }^{9.87}\right)$. Receiving referrals from a podiatrist was associated with sending referrals to a podiatrist $\left(O R_{\text {adj }} 10.04\right)$ and also being co-located with a podiatrist $\left(\mathrm{OR}_{\mathrm{adj}} 2.02\right)$.

\section{Conclusion}

This study provides initial evidence for the referral relationship between Australian osteopaths and podiatrists. Further work is now required to understand the nature of these referrals, including the complaints resulting in referral and outcomes of care. This information will be invaluable to those involved in health policy development and the professions advocating for their role in the wider healthcare system.

\section{Background}

Multidisciplinary care has been associated with benefits for patients and health professionals alike. These benefits include improved health outcomes, patient satisfaction, efficient use of resources, and job satisfaction for team members [1]. Working in a multidisciplinary care environment can be significantly influenced by context [2], particularly practice location and co-location with other health professionals [3, 4]. Health professionals located in tertiary care environments can readily engage in multidisciplinary care, given the ease of access to other health professionals. In the primary care environment, however, this 
immediacy of access may be more challenging to achieve and may require different pathways for patients to involve other health professionals in their care.

Australian podiatrists work in both public and private health contexts where they provide care for a range of lower limb conditions. In contrast, Australian osteopaths are predominantly located in private health care settings, where they provide care for a range of musculoskeletal complaints, including those affecting the lower limb [5-7]. Both professions are government registered in Australia, with accredited pre-registration programmes $[8,9]$. At the end of 2019 , there were 5,509 registered podiatrists, with nearly $60 \%$ identifying as female [10], and 2,723 registered osteopaths, with $55 \%$ identifying as female [11]. At the time over half of each the practitioners in each profession were less than 40 years of age. It is not possible to ascertain the practice location (public versus private) from this registration data; however, other works suggest that in Australia $90 \%$ of osteopaths [5] and approximately $72 \%$ of podiatrists [12] are located in private practice.

Australian podiatrists and osteopaths share several practice commonalities —in particular, the care of lower limb musculoskeletal complaints [5-7]. In Australia, services provided by both professions are included in the Medicare Chronic Disease Management (CDM) plan scheme [13], whereby patients can access, under Medicare, up to five allied health services per year to assist with the management of a chronic complaint, including musculoskeletal issues. This scheme entitles patients to a rebate from the Australian Government to assist with the costs of their care [13]. The CDM scheme presents an opportunity for osteopaths and podiatrists to work as part of a patient's multidisciplinary care team. Menz [14] reported that in the 2004-2008 period over 1.3 million consultations for podiatry care and 82,486 consultations with osteopaths were funded over the same period through the CDM scheme [15], with the rebates facilitated through the Medicare EasyClaim system. The almost ten-fold difference between the number of podiatry consultations and the number of osteopathy consultations through the CDM scheme is likely to be due to podiatrists having a narrower specialisation (foot care), and hence less competition compared with osteopaths, who are just one type of provider of musculoskeletal care under the CDM scheme.

Practice-based research networks (PBRNs) foster research, develop practice-relevant research questions, and assist knowledge translation to improve clinical care $[16,17]$. PBRNs have been used both in Australia and internationally across the medical and allied health professions [5, 16, 18-21]. The current study explored the demographic, practice and clinical-management characteristics of the practice of Australian osteopaths who send referrals to, and receive referrals from, podiatrists through the Osteopathy Research and Innovation Network (ORION) - the largest voluntary nationally representative PBRN in osteopathy worldwide [5, 22]. Little is known about the patterns of patient referrals between the osteopathy and podiatry professions in Australia. An emerging picture of referrals for both of these professions in Australia suggests that referrals are made to and from a range of health professionals [5, $6,23]$. The data from this secondary analysis of the ORION PBRN will not only contribute to our understanding of how these two professions work together in the Australian healthcare system but also 
inform interprofessional education in pre- and post-registration training programmes, and assist with the development of health policy for interprofessional care.

\section{Method}

Ethics approval for the current study was provided by the University of Technology Sydney (\#2014000759), and all participants in the study provided informed consent. All methods for this study were performed in accordance with the National Health and Medical Research Council guidelines. The study is a secondary analysis of data obtained from the ORION project (http://www.orion-arccim.com/).

\section{Sample}

Nine-hundred and ninety-two ( $N=992)$ responses were received, representing $49 \%$ of the osteopathy profession at time of completion in July_December 2016. Participants were required to be registered osteopaths practising in Australia. Study participants were recruited through the Australian osteopathy professional association (Osteopathy Australia) and by word of mouth. The baseline sample of participants (including that the sample is nationally representative on a number of key indicators) is extensively described elsewhere [5].

\section{Questionnaire}

A questionnaire was developed to establish ORION baseline data and this data is described elsewhere [5, 22]. Participants were invited to complete a self-report 27-item questionnaire designed to collect a range of demographic (e.g., age, gender, highest osteopathy degree, other degrees, length of time in clinical practice), clinical (e.g., presenting conditions, patient groups treated, techniques and adjuncts applied) and practice characteristics (e.g., patient care hours per week, visits per week, co-located with other health professionals, referral to and/or from other health professionals). The analysis presented in this paper focuses on the demographic, practice and clinical management characteristics of osteopaths associated with referrals to/from Australian podiatrists.

\section{Statistical analyses}

Participant responses were analysed based on whether the participant sent referrals or received referrals, which were treated independently as the outcome variable. Other variables were analysed in relation to these two independent variables, using independent t-tests and chi-square tests, as appropriate. Effect size and unadjusted odds ratios were calculated for statistically significant variables $(p<0.05)$. Analyses that reported $p<0.10$ were included in a subsequent backward logistic regression model to examine those variables that were statistically significantly associated with receiving or sending referrals to podiatrists. Adjusted odds ratios were estimated, and $95 \%$ confidence intervals were reported for 
significant variables. Alpha was set at 0.05. All descriptive statistical analyses, t-tests, chi-square tests and the backward regression modelling were performed using SPSS version 25 (IBM Corp., Armonk, NY, USA).

\section{Results}

Responses were received from all 992 osteopaths who responded to the ORION questionnaire questions regarding receiving referrals from a podiatrist (yes/no) and sending referrals to a podiatrist (yes/no), with no missing data. One hundred and forty-seven respondents ( $n=147,14.8 \%)$ reported being co-located with a podiatrist. Sending referrals to a podiatrist was reported by 651 participants $(65.6 \%)$ and receiving referrals from a podiatrist by 471 participants (47.5\%). The demographic characteristics of those who reported sending and/or receiving referrals are provided in Table 1. Females were significantly less likely than males to send referrals (OR 0.76), and osteopaths involved in clinical supervision were $60 \%$ more likely to receive referrals from podiatrists then were osteopaths who were not involved in clinical supervision (OR 1.60). 
Table 1

Practitioner characteristics of Australian osteopaths who report sending referrals to, and/or receiving referrals from, podiatrists.

\begin{tabular}{|c|c|c|c|c|c|c|c|c|}
\hline \multirow[b]{2}{*}{ Gender } & \multicolumn{4}{|c|}{ Send referrals } & \multicolumn{4}{|c|}{ Receive referrals } \\
\hline & $\begin{array}{l}\text { Yes } \\
(n=651)\end{array}$ & $\begin{array}{l}\text { No } \\
(n=341)\end{array}$ & $\begin{array}{l}\mathrm{p}- \\
\text { value }\end{array}$ & $\begin{array}{l}\text { Odds } \\
\text { ratio }\end{array}$ & $\begin{array}{l}\text { Yes } \\
(n=471)\end{array}$ & $\begin{array}{l}\text { No } \\
(n=521)\end{array}$ & $\begin{array}{l}\mathrm{p}- \\
\text { value }\end{array}$ & $\begin{array}{l}\text { Odds } \\
\text { ratio }\end{array}$ \\
\hline Male & $\begin{array}{l}393 \\
(60.4 \%)\end{array}$ & $\begin{array}{l}183 \\
(53.7 \%)\end{array}$ & & & $\begin{array}{l}257 \\
(25.9 \%)\end{array}$ & $\begin{array}{l}319 \\
(32.2 \%)\end{array}$ & & \\
\hline Female & $\begin{array}{l}258 \\
(39.6 \%)\end{array}$ & $\begin{array}{l}158 \\
(46.3 \%)\end{array}$ & 0.04 & $\begin{array}{l}0.76 \\
{[0.59} \\
0.99]\end{array}$ & $\begin{array}{l}376 \\
(42.4 \%)\end{array}$ & $\begin{array}{l}40 \\
(37.7 \%)\end{array}$ & 0.03 & $\begin{array}{l}1.35 \\
{[1.02,} \\
1.69]\end{array}$ \\
\hline
\end{tabular}

Age (years)

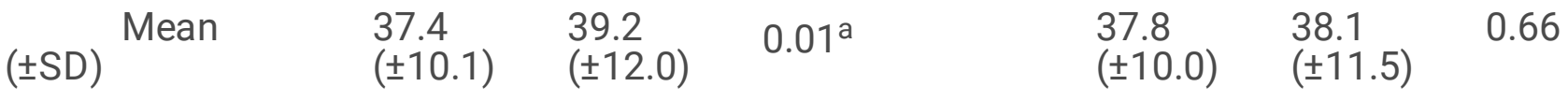

Years in clinical practice

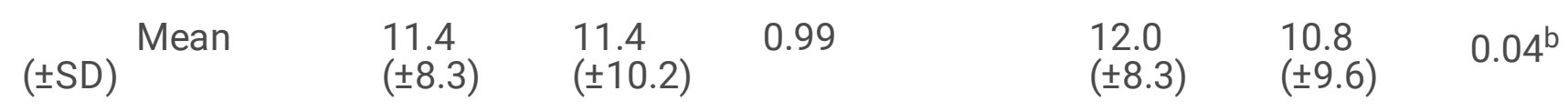

\section{Patient care hours per week}

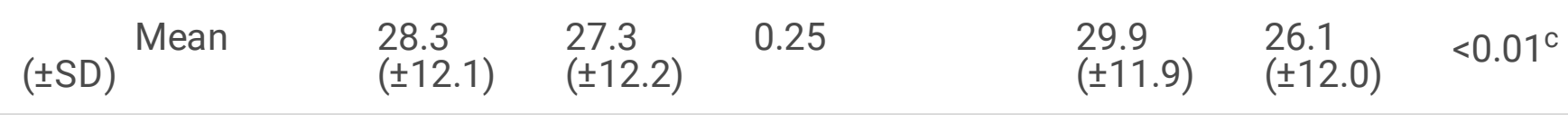

\section{Patient visits per week}

\begin{tabular}{|c|c|c|c|c|c|}
\hline$( \pm S D)$ Mean & $\begin{array}{l}37.3 \\
( \pm 18.9)\end{array}$ & $\begin{array}{l}35.0 \\
( \pm 18.0)\end{array}$ & 0.10 & $\begin{array}{l}40.8 \\
( \pm 19.6)\end{array}$ & $\begin{array}{l}32.6 \\
( \pm 16.8)\end{array}$ \\
\hline
\end{tabular}

\section{Qualification ( $n$, \\ \%)}

\begin{tabular}{cllllll|} 
Diploma & 36 & 26 & 0.34 & 29 & 33 & 0.89 \\
& $(5.5 \%)$ & $(7.6 \%)$ & $(2.9 \%)$ & $(3.3 \%)$ & \\
$\begin{array}{c}\text { Advanced } \\
\text { diploma }\end{array}$ & 5 & 4 & 5 & 4 \\
Bachelor & $(0.8 \%)$ & $(1.2 \%)$ & $(0.5 \%)$ & $(0.4 \%)$ \\
degree & $(22.1 \%)$ & $(21.7 \%)$ & 111 & 107 \\
Master's & 450 & 231 & $(11.2 \%)$ & $(10.8 \%)$ \\
degree & $(69.1 \%)$ & $(67.7 \%)$ & 316 & 365 \\
PhD & 2 & 3 & $(31.9 \%)$ & $(36.8 \%)$ \\
& $(0.3 \%)$ & $(0.9 \%)$ & 2 & 3 \\
Other & 14 & 3 & $(0.2 \%)$ & $(0.3 \%)$ \\
& $(2.2 \%)$ & $(0.9 \%)$ & 8 & 9 \\
& & & $(0.8 \%)$ & $(0.9 \%)$ \\
\hline
\end{tabular}




\begin{tabular}{|c|c|c|c|c|c|c|c|}
\hline $\begin{array}{l}\text { University } \\
\text { teaching }\end{array}$ & $\begin{array}{l}73 \\
(11.2 \%)\end{array}$ & $\begin{array}{l}43 \\
(12.6 \%)\end{array}$ & 0.52 & $\begin{array}{l}48 \\
(10.2 \%)\end{array}$ & $\begin{array}{l}68 \\
(13.1 \%)\end{array}$ & 0.16 & \\
\hline $\begin{array}{l}\text { Clinical } \\
\text { supervision }\end{array}$ & $\begin{array}{l}104 \\
(16.0 \%)\end{array}$ & $\begin{array}{l}46 \\
(13.5 \%)\end{array}$ & 0.30 & $\begin{array}{l}86 \\
(18.3 \%)\end{array}$ & $\begin{array}{l}64 \\
(12.3 \%)\end{array}$ & $<0.01$ & $\begin{array}{l}1.60 \\
{[1.12} \\
2.26]\end{array}$ \\
\hline $\begin{array}{l}\text { Professional } \\
\text { organisations }\end{array}$ & $\begin{array}{l}65 \\
(10.0 \%)\end{array}$ & $\begin{array}{l}42 \\
(12.3 \%)\end{array}$ & 0.26 & $\begin{array}{l}49 \\
(10.4 \%)\end{array}$ & $\begin{array}{l}58 \\
(11.1 \%)\end{array}$ & 0.71 & \\
\hline Research & $\begin{array}{l}35 \\
(5.4 \%)\end{array}$ & $\begin{array}{l}19 \\
(5.6 \%)\end{array}$ & 0.89 & $\begin{array}{l}23 \\
(4.9 \%)\end{array}$ & $\begin{array}{l}31 \\
(6.0 \%)\end{array}$ & 0.46 & \\
\hline Volunteer & $\begin{array}{l}110 \\
(16.9 \%)\end{array}$ & $\begin{array}{l}49 \\
(1.4 \%)\end{array}$ & 0.30 & $\begin{array}{l}80 \\
(17.0 \%)\end{array}$ & $\begin{array}{l}79 \\
(15.2 \%)\end{array}$ & 0.43 & \\
\hline
\end{tabular}

Osteopaths co-located with a medical specialist were less likely to send referrals to a podiatrist $(O R 0.48)$, than those who were co-located with a podiatrist (OR 3.08) or exercise physiologist (OR 2.27) (Table 2). Australian osteopaths who reported sending referrals to podiatrists were also more likely to send referrals to other osteopaths (OR 1.54) and more than twice as likely to send referrals to a range of medical, allied health and complementary medicine professionals (Table 2). Osteopaths who reported sending and receiving referrals to other health professionals were also more likely to send referrals to, and/or receive referrals from, podiatrists. Osteopaths who send referrals to podiatrists were more than seven times more likely to report using orthopaedic testing in patient assessment than osteopaths who do not send referrals to podiatrists, and were $30 \%$ more likely to use the Medicare EasyClaim system to claim rebates under the CDM scheme [13] (Table 2). 
Table 2

Practice characteristics of Australian osteopaths who report sending referrals to, and/or receiving referrals from, podiatrists.<

Send referrals to podiatrist

Yes No

$(n=651)$ $\underset{(n=341)}{\text { No }}$

$(n=341)$
Receive referrals from podiatrists

p- OR Yes

value $[95 \% \mathrm{Cl}]$

$(n=471)$
No

$(n=521)$ p- OR

value $(95 \% \mathrm{Cl})$

\section{Practice \\ location}

\begin{tabular}{|c|c|c|c|c|c|c|c|}
\hline practice & $\begin{array}{l}538 \\
(82.6 \%)\end{array}$ & $\begin{array}{l}282 \\
(82.7 \%)\end{array}$ & 0.98 & - & $\begin{array}{l}387 \\
(82.2 \%)\end{array}$ & $\begin{array}{l}433 \\
(83.1 \%)\end{array}$ & 0.70 \\
\hline \begin{tabular}{l}
\multicolumn{2}{c}{ More } \\
than one \\
practice \\
location
\end{tabular} & $\begin{array}{l}216 \\
(33.2 \%)\end{array}$ & $\begin{array}{l}131 \\
(38.4 \%)\end{array}$ & 0.10 & - & $\begin{array}{l}162 \\
(34.4 \%)\end{array}$ & $\begin{array}{l}185 \\
(35.5 \%)\end{array}$ & 0.71 \\
\hline
\end{tabular}

\section{Co-located with other}

health professionals ('yes')

\begin{tabular}{|c|c|c|c|c|c|c|c|}
\hline Osteopath & $\begin{array}{l}432 \\
(66.4 \%)\end{array}$ & $\begin{array}{l}211 \\
(61.9 \%)\end{array}$ & 0.16 & - & $\begin{array}{l}328 \\
(69.6 \%)\end{array}$ & $\begin{array}{l}315 \\
(60.5 \%)\end{array}$ & $<0.01$ \\
\hline
\end{tabular}

\begin{tabular}{|c|c|c|c|c|c|c|c|c|}
\hline $\begin{array}{l}\text { General } \\
\text { Practitioner }\end{array}$ & $\begin{array}{l}45 \\
(6.9 \%)\end{array}$ & $\begin{array}{l}27 \\
(7.9 \%)\end{array}$ & 0.56 & - & $\begin{array}{l}34 \\
(7.2 \%)\end{array}$ & $\begin{array}{l}38 \\
(7.3 \%)\end{array}$ & 0.96 & - \\
\hline $\begin{array}{l}\text { Specialist } \\
\text { Medical } \\
\text { Practitioner }\end{array}$ & $\begin{array}{l}15 \\
(2.3 \%)\end{array}$ & $\begin{array}{l}16 \\
(4.7 \%)\end{array}$ & 0.04 & $\begin{array}{l}0.48 \\
{[0.23} \\
0.98]\end{array}$ & $\begin{array}{l}14 \\
(3.0 \%)\end{array}$ & $\begin{array}{l}17 \\
(3.3 \%)\end{array}$ & 0.79 & - \\
\hline Podiatrist & $\begin{array}{l}123 \\
(18.9 \%)\end{array}$ & $\begin{array}{l}24 \\
(7.0 \%)\end{array}$ & $<0.01$ & $\begin{array}{l}3.08 \\
{[1.94} \\
4.87]\end{array}$ & $\begin{array}{l}102 \\
(21.7 \%)\end{array}$ & $\begin{array}{l}45 \\
(8.6 \%)\end{array}$ & $<0.01$ & $\begin{array}{l}2.92 \\
{[2.00,} \\
4.25]\end{array}$ \\
\hline Physiotherapist & $\begin{array}{l}100 \\
(15.4 \%)\end{array}$ & $\begin{array}{l}44 \\
(12.9 \%)\end{array}$ & 0.30 & - & $\begin{array}{l}71 \\
(15.1 \%)\end{array}$ & $\begin{array}{l}73 \\
(14.0 \%)\end{array}$ & 0.63 & - \\
\hline $\begin{array}{l}\text { Exercise } \\
\text { Physiologist }\end{array}$ & $\begin{array}{l}99 \\
(15.2 \%)\end{array}$ & $\begin{array}{l}25 \\
(7.3 \%)\end{array}$ & $<0.01$ & $\begin{array}{l}2.27 \\
{[1.43} \\
3.59]\end{array}$ & $\begin{array}{l}66 \\
(14.0 \%)\end{array}$ & $\begin{array}{l}58 \\
(11.1 \%)\end{array}$ & 0.17 & - \\
\hline $\begin{array}{l}\text { Occupational } \\
\text { Therapist }\end{array}$ & $\begin{array}{l}13 \\
(2.0 \%)\end{array}$ & $\begin{array}{l}6 \\
(1.8 \%)\end{array}$ & 0.79 & - & $\begin{array}{l}10 \\
(2.1 \%)\end{array}$ & $\begin{array}{l}9 \\
(1.0 \%)\end{array}$ & 0.65 & - \\
\hline Psychologist & $\begin{array}{l}120 \\
(18.4 \%)\end{array}$ & $\begin{array}{l}71 \\
(20.8 \%)\end{array}$ & 0.36 & - & $\begin{array}{l}89 \\
(18.9 \%)\end{array}$ & $\begin{array}{l}102 \\
(19.6 \%)\end{array}$ & 0.78 & - \\
\hline $\begin{array}{l}\text { Massage } \\
\text { Therapist }\end{array}$ & $\begin{array}{l}343 \\
(52.7 \%)\end{array}$ & $\begin{array}{l}158 \\
(46.3 \%)\end{array}$ & 0.06 & - & $\begin{array}{l}244 \\
(51.8 \%)\end{array}$ & $\begin{array}{l}257 \\
(49.3 \%)\end{array}$ & 0.43 & - \\
\hline Acupuncturist & $\begin{array}{l}116 \\
(17.8 \%)\end{array}$ & $\begin{array}{l}72 \\
(21.1 \%)\end{array}$ & 0.21 & - & $\begin{array}{l}79 \\
(16.8 \%)\end{array}$ & $\begin{array}{l}109 \\
(20.9 \%)\end{array}$ & 0.09 & - \\
\hline
\end{tabular}




\begin{tabular}{|c|c|c|c|c|c|c|c|c|}
\hline Naturopath & $\begin{array}{l}130 \\
(20.0 \%)\end{array}$ & $\begin{array}{l}63 \\
(18.5 \%)\end{array}$ & 0.57 & - & $\begin{array}{l}97 \\
(20.6 \%)\end{array}$ & $\begin{array}{l}96 \\
(18.4 \%)\end{array}$ & 0.39 & - \\
\hline Dietician & $\begin{array}{l}49 \\
(7.5 \%)\end{array}$ & $\begin{array}{l}23 \\
(6.7 \%)\end{array}$ & 0.65 & - & $\begin{array}{l}40 \\
(8.5 \%)\end{array}$ & $\begin{array}{l}32 \\
(6.1 \%)\end{array}$ & 0.15 & - \\
\hline Nutritionist & $\begin{array}{l}50 \\
(7.7 \%)\end{array}$ & $\begin{array}{l}28 \\
(8.2 \%)\end{array}$ & 0.77 & - & $\begin{array}{l}44 \\
(9.3 \%)\end{array}$ & $\begin{array}{l}34 \\
(6.5 \%)\end{array}$ & 0.10 & - \\
\hline \multicolumn{9}{|c|}{$\begin{array}{l}\text { Send referrals to other } \\
\text { health professionals ('yes') }\end{array}$} \\
\hline Osteopath & $\begin{array}{l}356 \\
(54.7 \%)\end{array}$ & $\begin{array}{l}150 \\
(44.0 \%)\end{array}$ & $<0.01$ & $\begin{array}{l}1.54 \\
{[1.18,} \\
2.00]\end{array}$ & $\begin{array}{l}254 \\
(53.9 \%)\end{array}$ & $\begin{array}{l}252 \\
(48.4 \%)\end{array}$ & 0.08 & - \\
\hline $\begin{array}{l}\text { General } \\
\text { Practitioner }\end{array}$ & $\begin{array}{l}613 \\
(94.2 \%)\end{array}$ & $\begin{array}{l}265 \\
(77.7 \%)\end{array}$ & $<0.01$ & $\begin{array}{l}4.62 \\
{[3.05} \\
7.01]\end{array}$ & $\begin{array}{l}438 \\
(93.0 \%)\end{array}$ & $\begin{array}{l}440 \\
(84.5 \%)\end{array}$ & $<0.01$ & $\begin{array}{l}2.44 \\
{[1.60} \\
3.74]\end{array}$ \\
\hline $\begin{array}{l}\text { Specialist } \\
\text { Medical } \\
\text { Practitioner }\end{array}$ & $\begin{array}{l}343 \\
(52.7 \%)\end{array}$ & $\begin{array}{l}100 \\
(29.3 \%)\end{array}$ & $<0.01$ & $\begin{array}{l}2.68 \\
{[2.03} \\
3.55]\end{array}$ & $\begin{array}{l}245 \\
(52.0 \%)\end{array}$ & $\begin{array}{l}198 \\
(38.0 \%)\end{array}$ & $<0.01$ & $\begin{array}{l}1.77 \\
{[1.37} \\
2.27]\end{array}$ \\
\hline Podiatrist & - & - & - & - & $\begin{array}{l}418 \\
(88.7 \%)\end{array}$ & $\begin{array}{l}233 \\
(44.7 \%)\end{array}$ & $<0.01$ & $\begin{array}{l}9.75 \\
{[6.98,} \\
13.61]\end{array}$ \\
\hline Physiotherapist & $\begin{array}{l}266 \\
(40.9 \%)\end{array}$ & $\begin{array}{l}65 \\
(19.1 \%)\end{array}$ & $<0.01$ & $\begin{array}{l}2.93 \\
{[2.15} \\
4.00]\end{array}$ & $\begin{array}{l}180 \\
(38.2 \%)\end{array}$ & $\begin{array}{l}151 \\
(29.0 \%)\end{array}$ & $<0.01$ & $\begin{array}{l}1.51 \\
{[1.16,} \\
1.98]\end{array}$ \\
\hline $\begin{array}{l}\text { Exercise } \\
\text { Physiologist }\end{array}$ & $\begin{array}{l}306 \\
(47.0 \%)\end{array}$ & $\begin{array}{l}92 \\
(27.0 \%)\end{array}$ & $<0.01$ & $\begin{array}{l}2.40 \\
{[1.80} \\
3.19]\end{array}$ & $\begin{array}{l}217 \\
(46.1 \%)\end{array}$ & $\begin{array}{l}181 \\
(34.7 \%)\end{array}$ & $<0.01$ & $\begin{array}{l}1.60 \\
{[1.24} \\
2.07]\end{array}$ \\
\hline $\begin{array}{l}\text { Occupational } \\
\text { Therapist }\end{array}$ & $\begin{array}{l}87 \\
(13.4 \%)\end{array}$ & $\begin{array}{l}19 \\
(5.6 \%)\end{array}$ & $<0.01$ & $\begin{array}{l}2.61 \\
{[1.56} \\
4.37]\end{array}$ & $\begin{array}{l}66 \\
(14.0 \%)\end{array}$ & $\begin{array}{l}40 \\
(7.7 \%)\end{array}$ & $<0.01$ & $\begin{array}{l}1.96 \\
{[1.29,} \\
2.96]\end{array}$ \\
\hline Psychologist & $\begin{array}{l}265 \\
(40.7 \%)\end{array}$ & $\begin{array}{l}84 \\
(24.6 \%)\end{array}$ & $<0.01$ & $\begin{array}{l}2.10 \\
{[1.57} \\
2.81]\end{array}$ & $\begin{array}{l}183 \\
(38.9 \%)\end{array}$ & $\begin{array}{l}166 \\
(31.9 \%)\end{array}$ & 0.02 & $\begin{array}{l}1.36 \\
{[1.04,} \\
1.76]\end{array}$ \\
\hline $\begin{array}{l}\text { Massage } \\
\text { Therapist }\end{array}$ & $\begin{array}{l}492 \\
(75.6 \%)\end{array}$ & $\begin{array}{l}179 \\
(52.5 \%)\end{array}$ & $<0.01$ & $\begin{array}{l}2.80 \\
{[2.12} \\
3.70]\end{array}$ & $\begin{array}{l}354 \\
(75.2 \%)\end{array}$ & $\begin{array}{l}317 \\
(60.8 \%)\end{array}$ & $<0.01$ & $\begin{array}{l}1.95 \\
{[1.48,} \\
2.56]\end{array}$ \\
\hline Acupuncturist & $\begin{array}{l}335 \\
(51.5 \%)\end{array}$ & $\begin{array}{l}116 \\
(34.0 \%)\end{array}$ & $<0.01$ & $\begin{array}{l}2.05 \\
{[1.56,} \\
2.70]\end{array}$ & $\begin{array}{l}241 \\
(51.2 \%)\end{array}$ & $\begin{array}{l}210 \\
(40.3 \%)\end{array}$ & $<0.01$ & $\begin{array}{l}1.55 \\
{[1.21,} \\
1.99]\end{array}$ \\
\hline Naturopath & $\begin{array}{l}356 \\
(54.7 \%)\end{array}$ & $\begin{array}{l}121 \\
(35.5 \%)\end{array}$ & $<0.01$ & $\begin{array}{l}2.19 \\
{[1.67} \\
2.87]\end{array}$ & $\begin{array}{l}258 \\
(54.8 \%)\end{array}$ & $\begin{array}{l}219 \\
(42.0 \%)\end{array}$ & $<0.01$ & $\begin{array}{l}1.67 \\
{[1.30} \\
2.15]\end{array}$ \\
\hline Dietician & $\begin{array}{l}136 \\
(20.9 \%)\end{array}$ & $\begin{array}{l}31 \\
(9.1 \%)\end{array}$ & $<0.01$ & $\begin{array}{l}2.64 \\
{[1.74}\end{array}$ & $\begin{array}{l}101 \\
(21.4 \%)\end{array}$ & $\begin{array}{l}66 \\
(12.7 \%)\end{array}$ & $<0.01$ & $\begin{array}{l}1.88 \\
{[1.34}\end{array}$ \\
\hline
\end{tabular}




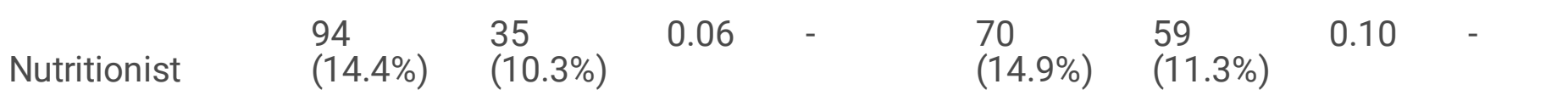

\section{Receive referrals from other health professionals ('yes')}

\begin{tabular}{|c|c|c|c|c|c|c|c|c|}
\hline Osteopath & $\begin{array}{l}429 \\
(65.9 \%)\end{array}$ & $\begin{array}{l}185 \\
(54.3 \%)\end{array}$ & $<0.01$ & $\begin{array}{l}1.63 \\
{[1.24,} \\
2.13]\end{array}$ & $\begin{array}{l}337 \\
(71.5 \%)\end{array}$ & $\begin{array}{l}277 \\
(53.2 \%)\end{array}$ & $<0.01$ & $\begin{array}{l}2.21 \\
{[1.70,} \\
2.88]\end{array}$ \\
\hline $\begin{array}{l}\text { General } \\
\text { Practitioner }\end{array}$ & $\begin{array}{l}594 \\
(91.2 \%)\end{array}$ & $\begin{array}{l}292 \\
(85.6 \%)\end{array}$ & $<0.01$ & $\begin{array}{l}1.75 \\
{[1.16,} \\
2.62]\end{array}$ & $\begin{array}{l}448 \\
(95.1 \%)\end{array}$ & $\begin{array}{l}438 \\
(84.1 \%)\end{array}$ & $<0.01$ & $\begin{array}{l}3.69 \\
{[2.28,} \\
5.97]\end{array}$ \\
\hline $\begin{array}{l}\text { Specialist } \\
\text { Medical } \\
\text { Practitioner }\end{array}$ & $\begin{array}{l}168 \\
(25.8 \%)\end{array}$ & $\begin{array}{l}69 \\
(20.2 \%)\end{array}$ & 0.05 & - & $\begin{array}{l}158 \\
(33.5 \%)\end{array}$ & $\begin{array}{l}79 \\
(15.2 \%)\end{array}$ & $<0.01$ & $\begin{array}{l}2.82 \\
{[2.08,} \\
3.84]\end{array}$ \\
\hline
\end{tabular}

$\begin{array}{lllll} & 418 & 53 & <0.01 & 9.75 \\ \text { Podiatrist } & (64.2 \%) & (15.5 \%) & & {[6.98,} \\ & & & 13.61]\end{array}$

$\begin{array}{lllllllll} & 190 & 76 & 0.02 & 1.44 & 165 & 101 & <0.01 & 2.24 \\ \text { Physiotherapist } & (29.2 \%) & (22.3 \%) & & {[1.06,} & (35.0 \%) & (19.4 \%) & & {[1.68,} \\ & & & & 1.95] & & & & 2.99]^{\prime}\end{array}$

$\begin{array}{lllllllll}\begin{array}{rlll}\text { Exercise } \\ \text { Physiologist }\end{array} & 196 & 62 & <0.01 & \begin{array}{l}1.94 \\ {[1.40,}\end{array} & \begin{array}{l}168 \\ (35.7 \%)\end{array} & \begin{array}{l}90 \\ (17.3 \%)\end{array} & <0.01 & 2.65 \\ & & & & 2.67]^{1} & & & & 3.97]^{\prime} \\ & 47 & 14.2 \%) & 0.05 & - & 46 & 15 & <0.01 & 3.65 \\ \begin{array}{l}\text { Occupational } \\ \text { Therapist }\end{array} & (7.2 \%) & (4.1 \%) & & & (9.8 \%) & (2.9 \%) & & {[2.01,} \\ & & & & & & & & 6.63]^{\prime}\end{array}$

\begin{tabular}{|c|c|c|c|c|c|c|c|c|}
\hline Psychologist & $\begin{array}{l}96 \\
(14.7 \%)\end{array}$ & $\begin{array}{l}58 \\
(17.0 \%)\end{array}$ & 0.35 & - & $\begin{array}{l}84 \\
(17.8 \%)\end{array}$ & $\begin{array}{l}70 \\
(13.4 \%)\end{array}$ & 0.05 & - \\
\hline $\begin{array}{l}\text { Massage } \\
\text { Therapist }\end{array}$ & $\begin{array}{l}529 \\
(81.3 \%)\end{array}$ & $\begin{array}{l}225 \\
(66.0 \%)\end{array}$ & $<0.01$ & $\begin{array}{l}2.23 \\
{[1.66,} \\
3.01]\end{array}$ & $\begin{array}{l}410 \\
(87.0 \%)\end{array}$ & $\begin{array}{l}344 \\
(66.0 \%)\end{array}$ & $<0.01$ & $\begin{array}{l}3.50 \\
{[2.50,} \\
4.78]\end{array}$ \\
\hline Acupuncturist & $\begin{array}{l}265 \\
(40.7 \%)\end{array}$ & $\begin{array}{l}105 \\
(30.8 \%)\end{array}$ & $<0.01$ & $\begin{array}{l}1.54 \\
{[1.17} \\
2.04]\end{array}$ & $\begin{array}{l}206 \\
(43.7 \%)\end{array}$ & $\begin{array}{l}164 \\
(31.5 \%)\end{array}$ & $<0.01$ & $\begin{array}{l}1.69 \\
{[1.30,} \\
2.19]\end{array}$ \\
\hline Naturopath & $\begin{array}{l}285 \\
(43.8 \%)\end{array}$ & $\begin{array}{l}115 \\
(33.7 \%)\end{array}$ & $<0.01$ & $\begin{array}{l}1.53 \\
{[1.16} \\
2.01]\end{array}$ & $\begin{array}{l}231 \\
(49.0 \%)\end{array}$ & $\begin{array}{l}169 \\
(32.4 \%)\end{array}$ & $<0.01$ & $\begin{array}{l}2.00 \\
{[1.55,} \\
2.59]\end{array}$ \\
\hline Dietician & $\begin{array}{l}28 \\
(4.3 \%)\end{array}$ & $\begin{array}{l}11 \\
(3.2 \%)\end{array}$ & 0.41 & - & $\begin{array}{l}29 \\
(6.2 \%)\end{array}$ & $\begin{array}{l}10 \\
(1.9 \%)\end{array}$ & $<0.01$ & $\begin{array}{l}3.35 \\
{[1.61,} \\
6.96]\end{array}$ \\
\hline Nutritic & $\begin{array}{l}37 \\
(5.7 \%)\end{array}$ & $\begin{array}{l}18 \\
(5.3 \%)\end{array}$ & 0.79 & - & $\begin{array}{l}39 \\
(8.3 \%)\end{array}$ & $\begin{array}{l}16 \\
(3.1 \%)\end{array}$ & $<0.01$ & $\begin{array}{l}2.85 \\
{[1.57,} \\
5.17]\end{array}$ \\
\hline
\end{tabular}


Diagnostic imaging

\begin{tabular}{|c|c|c|c|c|c|c|c|c|}
\hline $\begin{array}{l}\text { Referral } \\
\text { for imaging } \\
\text { ('often') }\end{array}$ & $\begin{array}{l}48 \\
(7.4 \%)\end{array}$ & $\begin{array}{l}25 \\
(7.3 \%)\end{array}$ & 0.98 & - & $\begin{array}{l}39 \\
(8.3 \%)\end{array}$ & $\begin{array}{l}34 \\
(6.5 \%)\end{array}$ & 0.29 & - \\
\hline $\begin{array}{l}\text { Investigation of } \\
\text { unknown } \\
\text { pathologies }\end{array}$ & $\begin{array}{l}496 \\
(76.2 \%)\end{array}$ & $\begin{array}{l}246 \\
(72.1 \%)\end{array}$ & 0.16 & - & $\begin{array}{l}370 \\
(78.6 \%)\end{array}$ & $\begin{array}{l}372 \\
(71.4 \%)\end{array}$ & 0.01 & $\begin{array}{l}1.47 \\
{[1.10,} \\
1.96]\end{array}$ \\
\hline $\begin{array}{l}\text { Investigation of } \\
\text { suspected } \\
\text { diagnosis }\end{array}$ & $\begin{array}{l}562 \\
(86.3 \%)\end{array}$ & $\begin{array}{l}273 \\
(80.1 \%)\end{array}$ & 0.01 & $\begin{array}{l}1.57 \\
{[1.11} \\
2.22]\end{array}$ & $\begin{array}{l}402 \\
(85.4 \%)\end{array}$ & $\begin{array}{l}433 \\
(83.1 \%)\end{array}$ & 0.33 & - \\
\hline $\begin{array}{l}\text { Investigation of } \\
\text { potential }\end{array}$ & $\begin{array}{l}496 \\
(76.2 \%)\end{array}$ & $\begin{array}{l}254 \\
(74.5 \%)\end{array}$ & 0.55 & - & $\begin{array}{l}373 \\
(79.2 \%)\end{array}$ & $\begin{array}{l}377 \\
(72.4 \%)\end{array}$ & 0.01 & $\begin{array}{l}1.45 \\
{[1.08,} \\
1.95]\end{array}$ \\
\hline
\end{tabular}

fractures

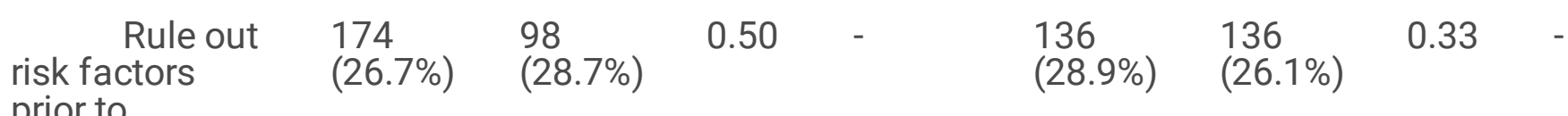

treatment

\begin{tabular}{|c|c|c|c|c|c|c|c|c|}
\hline $\begin{array}{l}\text { General } \\
\text { creening of the } \\
\text { jine }\end{array}$ & $\begin{array}{l}13 \\
(2.0 \%)\end{array}$ & $\begin{array}{l}19 \\
(5.6 \%)\end{array}$ & $<0.01$ & $\begin{array}{l}0.34 \\
{[0.17} \\
0.71]^{\prime}\end{array}$ & $\begin{array}{l}9 \\
(1.9 \%)\end{array}$ & $\begin{array}{l}23 \\
(4.4 \%)\end{array}$ & 0.03 & $\begin{array}{l}0.42 \\
{[0.19} \\
0.92]\end{array}$ \\
\hline
\end{tabular}

\section{Patient}

assessment

('yes')

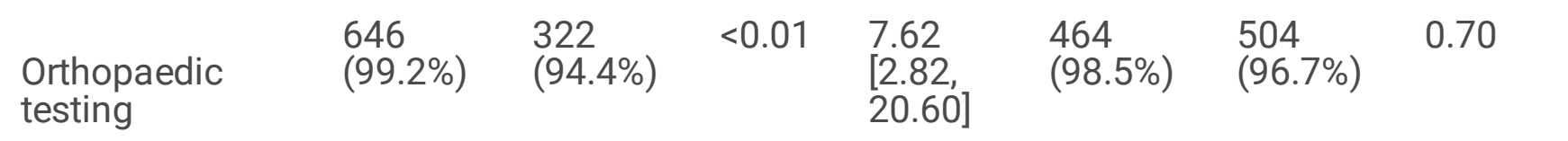

$\begin{array}{lllllllll}\begin{aligned} \text { Clinical } \\ \text { assessment }\end{aligned} & 320 & 148 & 0.08 & - & 234 & 234 & 0.13 & - \\ \text { algorithm } & (49.2 \%) & (43.4 \%) & & & (49.7 \%) & (44.9 \%) & & \end{array}$

$\begin{array}{lllllllll} & 611 & 307 & 0.03 & 1.69 & 442 & 476 & 0.14 & - \\ \begin{array}{l}\text { Neurological } \\ \text { testing }\end{array} & (93.9 \%) & (90.0 \%) & & {[1.05,} & (93.8 \%) & (91.4 \%) & & \\ & & & & 2.72] & & & \end{array}$

$\begin{array}{lllllllll} & 433 & 200 & 0.01 & 1.40 & 306 & 327 & 0.47 & - \\ \text { Screening } & (66.5 \%) & (58.7 \%) & & {[1.07,} & (65.0 \%) & (62.8 \%) & & \\ \text { questionnaire } & & & & 1.83] & & & \end{array}$

$\begin{array}{rlllllll}\text { Cranial } & 443 & 229 & 0.77 & - & 318 & 354 & 0.88 \\ \text { nerve testing } & (68.0 \%) & (6.72 \%) & & & (67.5 \%) & (67.9 \%) & \end{array}$

\section{Payment strategies}

$\begin{array}{lll}\text { HICAPS } & 603 & 301 \\ & (92.8 \%) & (88.8 \%)\end{array}$

$0.03 \quad 1.62$
$[1.03$




$\begin{array}{cllllllll}\text { Medicare } & 302 & 129 & 0.01 & 1.42 & 221 & 210 & 0.03 & 1.31 \\ \text { EasyClaim } & (46.4 \%) & (37.8 \%) & & {[1.09,} & (46.9 \%) & (40.3 \%) & & {[1.02} \\ & & & & 1.86] & & & & 1.68]\end{array}$

Australian osteopaths who reported sending referrals to, and/or receiving referrals from, podiatrists were $50 \%$ more likely to discuss physical activity and over $70 \%$ more likely to use sports taping as part of their patient management than their counterparts who did not send/receive podiatrist referrals (Table 3 ).

Osteopaths who reported receiving referrals from podiatrists were approximately $\mathrm{r} 30 \%$ more likely to treat patients over the age of 65 years than were osteopaths who do not receive referrals from podiatrists (Table 3). 
Table 3

Clinical management characteristics of Australian osteopaths who reported sending referrals to, and/or receiving referrals from, podiatrists.

Send referrals

Yes

$(n=651)$
Receive referrals

$\begin{array}{llllll}\text { p- } & \text { OR } & \text { Yes } & \text { No } & \text { p- } & \text { OR } \\ \text { value } & 95 \% & (n=471) & (n=521) & \text { value } & 95 \% \\ & {[C l]} & & & & {[C l]}\end{array}$

Discuss with

patients ('often')

\begin{tabular}{|c|c|c|c|c|c|c|c|c|}
\hline Diet & $\begin{array}{l}239 \\
(36.8 \%)\end{array}$ & $\begin{array}{l}136 \\
(39.9 \%)\end{array}$ & 0.34 & - & $\begin{array}{l}181 \\
(38.6 \%)\end{array}$ & $\begin{array}{l}194 \\
(37.2 \%)\end{array}$ & 0.66 & - \\
\hline $\begin{array}{l}\text { Smoking and } \\
\text { drug use }\end{array}$ & $\begin{array}{l}115 \\
(1.7 \%)\end{array}$ & $\begin{array}{l}64 \\
(18.8 \%)\end{array}$ & 0.68 & - & $\begin{array}{l}90 \\
(19.1 \%)\end{array}$ & $\begin{array}{l}89 \\
(17.1 \%)\end{array}$ & 0.41 & - \\
\hline $\begin{array}{l}\text { Physical } \\
\text { activity }\end{array}$ & $\begin{array}{l}591 \\
(90.9 \%)\end{array}$ & $\begin{array}{l}295 \\
(86.5 \%)\end{array}$ & 0.03 & $\begin{array}{l}1.56 \\
{[1.04,} \\
2.35]\end{array}$ & $\begin{array}{l}436 \\
(92.8 \%)\end{array}$ & $\begin{array}{l}450 \\
(86.4 \%)\end{array}$ & $<0.01$ & $\begin{array}{l}2.02 \\
{[1.32,} \\
3.11]\end{array}$ \\
\hline $\begin{array}{l}\text { Occupation } \\
\text { Health \& Safety }\end{array}$ & $\begin{array}{l}346 \\
(53.4 \%)\end{array}$ & $\begin{array}{l}160 \\
(46.9 \%)\end{array}$ & 0.05 & - & $\begin{array}{l}273 \\
(58.2 \%)\end{array}$ & $\begin{array}{l}233 \\
(44.8 \%)\end{array}$ & $<0.01$ & $\begin{array}{l}1.72 \\
{[1.33,} \\
2.21]\end{array}$ \\
\hline $\begin{array}{r}\text { Pain } \\
\text { counselling }\end{array}$ & $\begin{array}{l}175 \\
(26.9 \%)\end{array}$ & $\begin{array}{l}91 \\
(26.7 \%)\end{array}$ & 0.95 & - & $\begin{array}{l}122 \\
(26.0 \%)\end{array}$ & $\begin{array}{l}144 \\
(27.6 \%)\end{array}$ & 0.55 & - \\
\hline Stress & $\begin{array}{l}314 \\
(48.4 \%)\end{array}$ & $\begin{array}{l}175 \\
(51.5 \%)\end{array}$ & 0.35 & - & $\begin{array}{l}224 \\
(47.8 \%)\end{array}$ & $\begin{array}{l}265 \\
(51.0 \%)\end{array}$ & 0.31 & - \\
\hline Nutrition & $\begin{array}{l}158 \\
(24.3 \%)\end{array}$ & $\begin{array}{l}94 \\
(27.6 \%)\end{array}$ & 0.26 & - & $\begin{array}{l}111 \\
(23.6 \%)\end{array}$ & $\begin{array}{l}141 \\
(27.1 \%)\end{array}$ & 0.21 & - \\
\hline Medication & $\begin{array}{l}262 \\
(40.3 \%)\end{array}$ & $\begin{array}{l}129 \\
(37.9 \%)\end{array}$ & 0.47 & - & $\begin{array}{l}193 \\
(41.1 \%)\end{array}$ & $\begin{array}{l}198 \\
(38.1 \%)\end{array}$ & 0.34 & - \\
\hline
\end{tabular}

\section{Patient}

presentations

('often')

\begin{tabular}{|c|c|c|c|c|c|c|c|c|c|}
\hline & Neck pain & $\begin{array}{l}642 \\
(98.8 \%)\end{array}$ & $\begin{array}{l}329 \\
(96.5 \%)\end{array}$ & 0.01 & $\begin{array}{l}2.93 \\
{[1.19,} \\
7.23]\end{array}$ & $\begin{array}{l}465 \\
(98.9 \%)\end{array}$ & $\begin{array}{l}506 \\
(97.1 \%)\end{array}$ & 0.05 & - \\
\hline & Thoracic pain & $\begin{array}{l}600 \\
(92.3 \%)\end{array}$ & $\begin{array}{l}309 \\
(90.6 \%)\end{array}$ & 0.36 & - & $\begin{array}{l}443 \\
(94.3 \%)\end{array}$ & $\begin{array}{l}466 \\
(89.4 \%)\end{array}$ & $<0.01$ & $\begin{array}{l}1.93 \\
{[1.20} \\
3.12]\end{array}$ \\
\hline pain & Low back & $\begin{array}{l}645 \\
(99.2 \%)\end{array}$ & $\begin{array}{l}332 \\
(97.6 \%)\end{array}$ & 0.04 & $\begin{array}{l}3.11 \\
{[1.00,} \\
9.57]\end{array}$ & $\begin{array}{l}466 \\
(99.1 \%)\end{array}$ & $\begin{array}{l}511 \\
(98.3 \%)\end{array}$ & 0.22 & - \\
\hline $\begin{array}{l}\text { mus } \\
\text { pain }\end{array}$ & $\begin{array}{l}\text { Hip } \\
\text { دloskeletal }\end{array}$ & $\begin{array}{l}500 \\
(76.9 \%)\end{array}$ & $\begin{array}{l}244 \\
(71.8 \%)\end{array}$ & 0.08 & - & $\begin{array}{l}373 \\
(79.4 \%)\end{array}$ & $\begin{array}{l}371 \\
(71.3 \%)\end{array}$ & $<0.01$ & $\begin{array}{l}1.54 \\
{[1.15,} \\
2.07]\end{array}$ \\
\hline
\end{tabular}




\begin{tabular}{|c|c|c|c|c|c|c|c|c|}
\hline $\begin{array}{l}\text { Knee } \\
\text { musculoskeletal } \\
\text { pain }\end{array}$ & $\begin{array}{l}324 \\
(50.0 \%)\end{array}$ & $\begin{array}{l}167 \\
(49.1 \%)\end{array}$ & 0.79 & - & $\begin{array}{l}256 \\
(54.6 \%)\end{array}$ & $\begin{array}{l}235 \\
(45.3 \%)\end{array}$ & $<0.01$ & $\begin{array}{l}1.45 \\
{[1.13,} \\
1.87]\end{array}$ \\
\hline $\begin{array}{l}\text { Ankle } \\
\text { musculoskeletal } \\
\text { pain }\end{array}$ & $\begin{array}{l}226 \\
(34.9 \%)\end{array}$ & $\begin{array}{l}107 \\
(31.4 \%)\end{array}$ & 0.27 & - & $\begin{array}{l}175 \\
(37.3 \%)\end{array}$ & $\begin{array}{l}158 \\
(30.4 \%)\end{array}$ & 0.02 & $\begin{array}{l}1.36 \\
{[1.05,} \\
1.78]\end{array}$ \\
\hline $\begin{array}{l}\text { Foot } \\
\text { musculoskeletal } \\
\text { pain }\end{array}$ & $\begin{array}{l}197 \\
(30.4 \%)\end{array}$ & $\begin{array}{l}97 \\
(28.4 \%)\end{array}$ & 0.53 & - & $\begin{array}{l}150 \\
(31.9 \%)\end{array}$ & $\begin{array}{l}144 \\
(27.7 \%)\end{array}$ & 0.15 & - \\
\hline $\begin{array}{l}\text { Shoulder } \\
\text { musculoskeletal } \\
\text { pain }\end{array}$ & $\begin{array}{l}539 \\
(83.1 \%)\end{array}$ & $\begin{array}{l}262 \\
(77.1 \%)\end{array}$ & 0.02 & $\begin{array}{l}1.46 \\
{[1.05} \\
2.02]\end{array}$ & $\begin{array}{l}407 \\
(86.6 \%)\end{array}$ & $\begin{array}{l}394 \\
(75.9 \%)\end{array}$ & $<0.01$ & $\begin{array}{l}2.05 \\
{[1.46,} \\
2.86]\end{array}$ \\
\hline $\begin{array}{l}\text { Elbow } \\
\text { musculoskeletal } \\
\text { pain }\end{array}$ & $\begin{array}{l}164 \\
(25.4 \%)\end{array}$ & $\begin{array}{l}87 \\
(25.6 \%)\end{array}$ & 0.94 & - & $\begin{array}{l}140 \\
(30.0 \%)\end{array}$ & $\begin{array}{l}111 \\
(21.4 \%)\end{array}$ & $<0.01$ & $\begin{array}{l}1.57 \\
{[1.78,} \\
2.10]\end{array}$ \\
\hline \begin{tabular}{l}
\multicolumn{1}{c}{ Wrist } \\
musculoskeletal \\
pain
\end{tabular} & $\begin{array}{l}122 \\
(18.8 \%)\end{array}$ & $\begin{array}{l}66 \\
(19.4 \%)\end{array}$ & 0.84 & - & $\begin{array}{l}92 \\
(19.6 \%)\end{array}$ & $\begin{array}{l}96 \\
(18.5 \%)\end{array}$ & 0.66 & - \\
\hline \begin{tabular}{l}
\multicolumn{1}{c}{ Hand } \\
musculoskeletal \\
pain
\end{tabular} & $\begin{array}{l}74 \\
(11.5 \%)\end{array}$ & $\begin{array}{l}47 \\
(13.9 \%)\end{array}$ & 0.27 & - & $\begin{array}{l}59 \\
(12.6 \%)\end{array}$ & $\begin{array}{l}62 \\
(12.0 \%)\end{array}$ & 0.80 & - \\
\hline $\begin{array}{l}\text { Postural } \\
\text { disorders }\end{array}$ & $\begin{array}{l}469 \\
(72.4 \%)\end{array}$ & $\begin{array}{l}206 \\
(60.4 \%)\end{array}$ & $<0.01$ & $\begin{array}{l}1.71 \\
{[1.03} \\
2.26]\end{array}$ & $\begin{array}{l}347 \\
(73.8 \%)\end{array}$ & $\begin{array}{l}328 \\
(63.2 \%)\end{array}$ & $<0.01$ & $\begin{array}{l}1.64 \\
{[1.25,} \\
2.16]\end{array}$ \\
\hline $\begin{array}{l}\text { Degenerative } \\
\text { spine conditions }\end{array}$ & $\begin{array}{l}411 \\
(63.4 \%)\end{array}$ & $\begin{array}{l}188 \\
(55.1 \%)\end{array}$ & 0.01 & $\begin{array}{l}1.41 \\
{[1.08} \\
1.84]\end{array}$ & $\begin{array}{l}319 \\
(67.9 \%)\end{array}$ & $\begin{array}{l}280 \\
(53.9 \%)\end{array}$ & $<0.01$ & $\begin{array}{l}1.80 \\
{[1.39,} \\
2.34]\end{array}$ \\
\hline $\begin{array}{l}\text { Headache } \\
\text { disorders }\end{array}$ & $\begin{array}{l}673 \\
(68.0 \%)\end{array}$ & $\begin{array}{l}219 \\
(22.1 \%)\end{array}$ & 0.43 & - & $\begin{array}{l}429 \\
(91.3 \%)\end{array}$ & $\begin{array}{l}463 \\
(89.0 \%)\end{array}$ & 0.24 & - \\
\hline $\begin{array}{l}\text { Migraine } \\
\text { disorders }\end{array}$ & $\begin{array}{l}264 \\
(40.7 \%)\end{array}$ & $\begin{array}{l}136 \\
(40.0 \%)\end{array}$ & 0.82 & - & $\begin{array}{l}199 \\
(42.3 \%)\end{array}$ & $\begin{array}{l}201 \\
(38.8 \%)\end{array}$ & 0.26 & - \\
\hline $\begin{array}{l}\text { Spine health } \\
\text { maintenance }\end{array}$ & $\begin{array}{l}303 \\
(46.8 \%)\end{array}$ & $\begin{array}{l}155 \\
(45.5 \%)\end{array}$ & 0.68 & - & $\begin{array}{l}224 \\
(47.8 \%)\end{array}$ & $\begin{array}{l}234 \\
(45.1 \%)\end{array}$ & 0.40 & - \\
\hline $\begin{array}{l}\text { Chronic or } \\
\text { persistent pain }\end{array}$ & $\begin{array}{l}405 \\
(62.5 \%)\end{array}$ & $\begin{array}{l}225 \\
(66.0 \%)\end{array}$ & 0.28 & - & $\begin{array}{l}299 \\
(63.8 \%)\end{array}$ & $\begin{array}{l}331 \\
(63.7 \%)\end{array}$ & 0.97 & - \\
\hline Tendinopathies & $\begin{array}{l}286 \\
(44.1 \%)\end{array}$ & $\begin{array}{l}124 \\
(36.4 \%)\end{array}$ & 0.02 & $\begin{array}{l}1.38 \\
{[1.06} \\
1.81]\end{array}$ & $\begin{array}{l}220 \\
(46.9 \%)\end{array}$ & $\begin{array}{l}190 \\
(36.5 \%)\end{array}$ & $<0.01$ & $\begin{array}{l}1.53 \\
{[1.19,} \\
1.98]\end{array}$ \\
\hline $\begin{array}{l}\text { Temporomandibular } \\
\text { joint disorders }\end{array}$ & $\begin{array}{l}115 \\
(17.7 \%)\end{array}$ & $\begin{array}{l}68 \\
(20.1 \%)\end{array}$ & 0.37 & - & $\begin{array}{l}87 \\
(18.6 \%)\end{array}$ & $\begin{array}{l}96 \\
(18.5 \%)\end{array}$ & 0.98 & - \\
\hline $\begin{array}{l}\text { Non- } \\
\text { musculoskeletal } \\
\text { disorders }\end{array}$ & $\begin{array}{l}65 \\
(10.1 \%)\end{array}$ & $\begin{array}{l}61 \\
(18.0 \%)\end{array}$ & $<0.01$ & $\begin{array}{l}0.51 \\
{[0.35} \\
0.75]\end{array}$ & $\begin{array}{l}59 \\
(12.6 \%)\end{array}$ & $\begin{array}{l}67 \\
(13.1 \%)\end{array}$ & 0.84 & - \\
\hline
\end{tabular}


Patient subgroups (treat 'often')

\begin{tabular}{|c|c|c|c|c|c|c|c|c|}
\hline of age Up to 3 years & $\begin{array}{l}96 \\
(14.8 \%)\end{array}$ & $\begin{array}{l}60 \\
(17.6 \%)\end{array}$ & 0.24 & - & $\begin{array}{l}78 \\
(16.7 \%)\end{array}$ & $\begin{array}{l}78 \\
(15.0 \%)\end{array}$ & 0.47 & - \\
\hline of age 4 to 18 years & $\begin{array}{l}186 \\
(28.6 \%)\end{array}$ & $\begin{array}{l}84 \\
(24.6 \%)\end{array}$ & 0.18 & - & $\begin{array}{l}148 \\
(31.5 \%)\end{array}$ & $\begin{array}{l}122 \\
(23.4 \%)\end{array}$ & $<0.01$ & $\begin{array}{l}1.50 \\
{[1.13,} \\
1.99]\end{array}$ \\
\hline of age ${ }^{\text {Over } 65 \text { years }}$ & $\begin{array}{l}386 \\
(59.4 \%)\end{array}$ & $\begin{array}{l}186 \\
(54.5 \%)\end{array}$ & 0.14 & - & $\begin{array}{l}288 \\
(61.3 \%)\end{array}$ & $\begin{array}{l}284 \\
(54.5 \%)\end{array}$ & 0.03 & $\begin{array}{l}1.32 \\
{[1.02,} \\
1.70]\end{array}$ \\
\hline $\begin{array}{l}\text { Aboriginal \& } \\
\text { Torres Strait Islander } \\
\text { peoples }\end{array}$ & $\begin{array}{l}7 \\
(1.1 \%)\end{array}$ & $0(0 \%)$ & 0.05 & - & $\begin{array}{l}4 \\
(0.9 \%)\end{array}$ & $\begin{array}{l}3 \\
(0.6 \%)\end{array}$ & 0.61 & - \\
\hline Pregnancy & $\begin{array}{l}237 \\
(36.5 \%)\end{array}$ & $\begin{array}{l}107 \\
(31.4 \%)\end{array}$ & 0.11 & - & $\begin{array}{l}187 \\
(39.8 \%)\end{array}$ & $\begin{array}{l}157 \\
(30.1 \%)\end{array}$ & $<0.01$ & $\begin{array}{l}1.53 \\
{[1.18,} \\
1.99]\end{array}$ \\
\hline $\begin{array}{l}\text { Non-English } \\
\text { speaking }\end{array}$ & $\begin{array}{l}24 \\
(3.7 \%)\end{array}$ & $\begin{array}{l}9 \\
(2.6 \%)\end{array}$ & 0.38 & - & $\begin{array}{l}14 \\
(3.0 \%)\end{array}$ & $\begin{array}{l}19 \\
(3.7 \%)\end{array}$ & 0.56 & - \\
\hline Sport injuries & $\begin{array}{l}350 \\
(53.8 \%)\end{array}$ & $\begin{array}{l}151 \\
(44.4 \%)\end{array}$ & $<0.01$ & $\begin{array}{l}1.46 \\
{[1.12} \\
1.90]\end{array}$ & $\begin{array}{l}271 \\
(57.7 \%)\end{array}$ & $\begin{array}{l}230 \\
(44.2 \%)\end{array}$ & $<0.01$ & $\begin{array}{l}1.72 \\
{[1.33} \\
2.21]\end{array}$ \\
\hline $\begin{array}{l}\text { Worker injury } \\
\text { (compensable) }\end{array}$ & $\begin{array}{l}68 \\
(10.5 \%)\end{array}$ & $\begin{array}{l}35 \\
(10.3 \%)\end{array}$ & 0.91 & - & $\begin{array}{l}52 \\
(11.1 \%)\end{array}$ & $\begin{array}{l}51 \\
(9.8 \%)\end{array}$ & 0.50 & - \\
\hline $\begin{array}{c}\text { Work injury } \\
\text { (non-compensable) }\end{array}$ & $\begin{array}{l}238 \\
(36.6 \%)\end{array}$ & $\begin{array}{l}103 \\
(30.2 \%)\end{array}$ & 0.04 & $\begin{array}{l}1.33 \\
{[1.01} \\
1.77]\end{array}$ & $\begin{array}{l}177 \\
(37.7 \%)\end{array}$ & $\begin{array}{l}164 \\
(31.5 \%)\end{array}$ & 0.04 & $\begin{array}{l}1.31 \\
{[1.01,} \\
1.71]\end{array}$ \\
\hline $\begin{array}{l}\text { Traffic injury } \\
\text { (compensable) }\end{array}$ & $\begin{array}{l}36 \\
(5.6 \%)\end{array}$ & $\begin{array}{l}18 \\
(5.3 \%)\end{array}$ & 0.85 & - & $\begin{array}{l}26 \\
(5.5 \%)\end{array}$ & $\begin{array}{l}28 \\
(5.4 \%)\end{array}$ & 0.92 & - \\
\hline $\begin{array}{r}\text { Traffic injury } \\
\text { (non-compensable) }\end{array}$ & $\begin{array}{l}73 \\
(11.2 \%)\end{array}$ & $\begin{array}{l}41 \\
(12.1 \%)\end{array}$ & 0.68 & - & $\begin{array}{l}56 \\
(11.9 \%)\end{array}$ & $\begin{array}{l}58 \\
(11.2 \%)\end{array}$ & 0.71 & - \\
\hline Post-surgery & $\begin{array}{l}52 \\
(8.0 \%)\end{array}$ & $\begin{array}{l}27 \\
(8.0 \%)\end{array}$ & 0.98 & - & $\begin{array}{l}47 \\
(10.0 \%)\end{array}$ & $\begin{array}{l}32 \\
(6.2 \%)\end{array}$ & 0.02 & $\begin{array}{l}1.70 \\
{[1.06} \\
2.71]\end{array}$ \\
\hline
\end{tabular}

Manual therapy (use 'often')

\begin{tabular}{|c|c|c|c|c|c|c|c|c|}
\hline Counterstrain & $\begin{array}{l}293 \\
(45.1 \%)\end{array}$ & $\begin{array}{l}127 \\
(37.2 \%)\end{array}$ & 0.01 & $\begin{array}{l}1.38 \\
{[1.06} \\
1.81]\end{array}$ & $\begin{array}{l}205 \\
(43.7 \%)\end{array}$ & $\begin{array}{l}215 \\
(41.3 \%)\end{array}$ & 0.43 & - \\
\hline $\begin{array}{c}\text { Muscle } \\
\text { energy technique }\end{array}$ & $\begin{array}{l}546 \\
(84.0 \%)\end{array}$ & $\begin{array}{l}242 \\
(71.0 \%)\end{array}$ & $<0.01$ & $\begin{array}{l}2.15 \\
{[1.57} \\
2.94]\end{array}$ & $\begin{array}{l}387 \\
(82.3 \%)\end{array}$ & $\begin{array}{l}401 \\
(77.0 \%)\end{array}$ & 0.03 & $\begin{array}{l}1.39 \\
{[1.02} \\
1.91]\end{array}$ \\
\hline $\begin{array}{l}\text { High-velocity, } \\
\text { low-amplitude } \\
\text { manipulation }\end{array}$ & $\begin{array}{l}441 \\
(67.8 \%)\end{array}$ & $\begin{array}{l}191 \\
(56.0 \%)\end{array}$ & $<0.01$ & $\begin{array}{l}1.65 \\
{[1.26,} \\
2.17]\end{array}$ & $\begin{array}{l}324 \\
(68.9 \%)\end{array}$ & $\begin{array}{l}308 \\
(59.1 \%)\end{array}$ & $<0.01$ & $\begin{array}{l}1.53 \\
{[1.18,} \\
1.99]\end{array}$ \\
\hline
\end{tabular}




\begin{tabular}{|c|c|c|c|c|c|c|c|c|}
\hline $\begin{array}{c}\text { Joint } \\
\text { manipulation }\end{array}$ & $\begin{array}{l}281 \\
(43.4 \%)\end{array}$ & $\begin{array}{l}112 \\
(32.8 \%)\end{array}$ & $<0.01$ & $\begin{array}{l}1.56 \\
{[1.19} \\
2.06]\end{array}$ & $\begin{array}{l}205 \\
(43.7 \%)\end{array}$ & $\begin{array}{l}188 \\
(36.2 \%)\end{array}$ & 0.01 & $\begin{array}{l}1.37 \\
{[1.06,} \\
1.77]\end{array}$ \\
\hline $\begin{array}{l}\text { Soft tissue } \\
\text { technique }\end{array}$ & $\begin{array}{l}584 \\
(90.0 \%)\end{array}$ & $\begin{array}{l}264 \\
(77.4 \%)\end{array}$ & $<0.01$ & $\begin{array}{l}2.62 \\
{[1.83} \\
3.76]\end{array}$ & $\begin{array}{l}422 \\
(90.0 \%)\end{array}$ & $\begin{array}{l}426 \\
(81.8 \%)\end{array}$ & $<0.01$ & $\begin{array}{l}2.00 \\
{[1.38,} \\
2.91]\end{array}$ \\
\hline release ${ }^{\text {Myofascial }}$ & $\begin{array}{l}426 \\
(65.6 \%)\end{array}$ & $\begin{array}{l}186 \\
(54.5 \%)\end{array}$ & $<0.01$ & $\begin{array}{l}1.59 \\
{[1.22} \\
2.09]\end{array}$ & $\begin{array}{l}312 \\
(66.4 \%)\end{array}$ & $\begin{array}{l}300 \\
(57.7 \%)\end{array}$ & $<0.01$ & $\begin{array}{l}1.45 \\
{[1.11,} \\
1.87]\end{array}$ \\
\hline $\begin{array}{l}\text { Visceral } \\
\text { techniques }\end{array}$ & $\begin{array}{l}56 \\
(8.6 \%)\end{array}$ & $\begin{array}{l}42 \\
(12.3 \%)\end{array}$ & 0.06 & - & $\begin{array}{l}46 \\
(9.8 \%)\end{array}$ & $\begin{array}{l}52 \\
(10.0 \%)\end{array}$ & 0.92 & - \\
\hline pump Lymphatic & $\begin{array}{l}52 \\
(8.0 \%)\end{array}$ & $\begin{array}{l}32 \\
(9.4 \%)\end{array}$ & 0.46 & - & $\begin{array}{l}35 \\
(7.4 \%)\end{array}$ & $\begin{array}{l}49 \\
(9.4 \%)\end{array}$ & 0.27 & - \\
\hline $\begin{array}{l}\text { Autonomic } \\
\text { balancing }\end{array}$ & $\begin{array}{l}93 \\
(14.3 \%)\end{array}$ & $\begin{array}{l}64 \\
(18.8 \%)\end{array}$ & 0.06 & - & $\begin{array}{l}68 \\
(14.5 \%)\end{array}$ & $\begin{array}{l}89 \\
(17.1 \%)\end{array}$ & 0.25 & - \\
\hline Biodynamics & $\begin{array}{l}88 \\
(13.5 \%)\end{array}$ & $\begin{array}{l}67 \\
(19.6 \%)\end{array}$ & 0.01 & - & $\begin{array}{l}69 \\
(14.7 \%)\end{array}$ & $\begin{array}{l}86 \\
(16.5 \%)\end{array}$ & 0.43 & - \\
\hline $\begin{array}{l}\text { Functional } \\
\text { technique }\end{array}$ & $\begin{array}{l}176 \\
(27.1 \%)\end{array}$ & $\begin{array}{l}94 \\
(27.6 \%)\end{array}$ & 0.87 & - & $\begin{array}{l}126 \\
(26.8 \%)\end{array}$ & $\begin{array}{l}144 \\
(27.6 \%)\end{array}$ & 0.77 & - \\
\hline $\begin{array}{c}\text { Balanced } \\
\text { ligamentous tension }\end{array}$ & $\begin{array}{l}216 \\
(33.2 \%)\end{array}$ & $\begin{array}{l}133 \\
(39.0 \%)\end{array}$ & 0.07 & - & $\begin{array}{l}155 \\
(33.0 \%)\end{array}$ & $\begin{array}{l}194 \\
(37.2 \%)\end{array}$ & 0.16 & - \\
\hline $\begin{array}{l}\text { Chapman's } \\
\text { reflexes }\end{array}$ & $\begin{array}{l}16 \\
(2.5 \%)\end{array}$ & $\begin{array}{l}8 \\
(2.4 \%)\end{array}$ & 0.91 & - & $\begin{array}{l}12 \\
(2.6 \%)\end{array}$ & $\begin{array}{l}12 \\
(2.3 \%)\end{array}$ & 0.80 & - \\
\hline $\begin{array}{l}\text { Trigger point } \\
\text { therapy }\end{array}$ & $\begin{array}{l}199 \\
(30.6 \%)\end{array}$ & $\begin{array}{l}59 \\
(17.4 \%)\end{array}$ & $<0.01$ & - & $\begin{array}{l}146 \\
(31.1 \%)\end{array}$ & $\begin{array}{l}112 \\
(21.5 \%)\end{array}$ & $<0.01$ & $\begin{array}{l}1.64 \\
{[1.23,} \\
2.18]\end{array}$ \\
\hline $\begin{array}{l}\text { Osteopathy in } \\
\text { the Cranial Field }\end{array}$ & $\begin{array}{l}141 \\
(21.7 \%)\end{array}$ & $\begin{array}{l}92 \\
(27.0 \%)\end{array}$ & 0.06 & - & $\begin{array}{l}99 \\
(21.1 \%)\end{array}$ & $\begin{array}{l}134 \\
(25.7 \%)\end{array}$ & 0.09 & - \\
\hline $\begin{array}{r}\text { Facilitated } \\
\text { positional release }\end{array}$ & $\begin{array}{l}115 \\
(17.7 \%)\end{array}$ & $\begin{array}{l}51 \\
(15.0 \%)\end{array}$ & 0.29 & - & $\begin{array}{l}84 \\
(17.9 \%)\end{array}$ & $\begin{array}{l}82 \\
(15.8 \%)\end{array}$ & 0.38 & - \\
\hline Dry needling & $\begin{array}{l}170 \\
(26.2 \%)\end{array}$ & $\begin{array}{l}64 \\
(18.8 \%)\end{array}$ & 0.01 & $\begin{array}{l}1.52 \\
{[1.10,} \\
2.11]\end{array}$ & $\begin{array}{l}121 \\
(25.7 \%)\end{array}$ & $\begin{array}{l}113 \\
(21.7 \%)\end{array}$ & 0.14 & - \\
\hline $\begin{array}{l}\text { Exercise } \\
\text { prescription }\end{array}$ & $\begin{array}{l}500 \\
(77.0 \%)\end{array}$ & $\begin{array}{l}233 \\
(68.3 \%)\end{array}$ & $<0.01$ & $\begin{array}{l}1.55 \\
{[1.16} \\
2.08]\end{array}$ & $\begin{array}{l}364 \\
(77.6 \%)\end{array}$ & $\begin{array}{l}369 \\
(70.8 \%)\end{array}$ & 0.01 & $\begin{array}{l}1.43 \\
\text { [1.07, } \\
1.90]\end{array}$ \\
\hline $\begin{array}{l}\text { Shockwave } \\
\text { therapy }\end{array}$ & $\begin{array}{l}11 \\
(1.7 \%)\end{array}$ & $\begin{array}{l}7 \\
(2.1 \%)\end{array}$ & 0.68 & - & $\begin{array}{l}8 \\
(1.7 \%)\end{array}$ & $\begin{array}{l}10 \\
(1.9 \%)\end{array}$ & 0.79 & - \\
\hline Ultrasound & $\begin{array}{l}14 \\
(2.2 \%)\end{array}$ & $\begin{array}{l}13 \\
(3.8 \%)\end{array}$ & 0.13 & - & $\begin{array}{l}13 \\
(2.8 \%)\end{array}$ & $\begin{array}{l}14 \\
(2.7 \%)\end{array}$ & 0.95 & - \\
\hline TENS & $\begin{array}{l}7 \\
(1.1 \%)\end{array}$ & $\begin{array}{l}12 \\
(3.5 \%)\end{array}$ & $<0.01$ & $\begin{array}{l}0.29 \\
{[0.11}\end{array}$ & $\begin{array}{l}5 \\
(1.1 \%)\end{array}$ & $\begin{array}{l}14 \\
(2.7 \%)\end{array}$ & 0.06 & - \\
\hline
\end{tabular}




\begin{tabular}{|c|c|c|c|c|c|c|c|c|}
\hline $\begin{array}{c}\text { Instrument } \\
\text { manipulation }\end{array}$ & $0(0 \%)$ & $\begin{array}{l}2 \\
(0.6 \%)\end{array}$ & 0.05 & - & $0(0 \%)$ & $\begin{array}{l}2 \\
(0.4 \%)\end{array}$ & 0.18 & - \\
\hline $\begin{array}{l}\text { Instrument } \\
\text { soft tissue }\end{array}$ & $\begin{array}{l}8 \\
(1.2 \%)\end{array}$ & $\begin{array}{l}4 \\
(1.2 \%)\end{array}$ & 0.94 & - & $\begin{array}{l}7 \\
(1.5 \%)\end{array}$ & $\begin{array}{l}5 \\
(1.0 \%)\end{array}$ & 0.45 & - \\
\hline Sport taping & $\begin{array}{l}93 \\
(14.3 \%)\end{array}$ & $\begin{array}{l}29 \\
(8.5 \%)\end{array}$ & $<0.01$ & $\begin{array}{l}1.79 \\
{[1.15,} \\
2.79]\end{array}$ & $\begin{array}{l}73 \\
(15.5 \%)\end{array}$ & $\begin{array}{l}49 \\
(9.4 \%)\end{array}$ & $<0.01$ & $\begin{array}{l}1.76 \\
{[1.02} \\
2.60]\end{array}$ \\
\hline
\end{tabular}

\section{Expanded practice scope ('definitely')}

\begin{tabular}{|c|c|c|c|c|c|c|c|c|}
\hline rights & $\begin{array}{l}175 \\
(26.9 \%)\end{array}$ & $\begin{array}{l}82 \\
(24.0 \%)\end{array}$ & 0.32 & - & $\begin{array}{l}128 \\
(27.2 \%)\end{array}$ & $\begin{array}{l}129 \\
(24.8 \%)\end{array}$ & 0.37 & - \\
\hline $\begin{array}{l}\qquad \text { Referral } \\
\text { rights to orthopaedic } \\
\text { surgeon }\end{array}$ & $\begin{array}{l}475 \\
(73.1 \%)\end{array}$ & $\begin{array}{l}228 \\
(66.9 \%)\end{array}$ & 0.04 & $\begin{array}{l}1.34 \\
{[1.01} \\
1.79]\end{array}$ & $\begin{array}{l}349 \\
(74.3 \%)\end{array}$ & $\begin{array}{l}354 \\
(67.9 \%)\end{array}$ & 0.03 & $\begin{array}{l}1.36 \\
{[1.03} \\
1.79]\end{array}$ \\
\hline $\begin{array}{l}\quad \text { Referral } \\
\text { rights to } \\
\text { paediatrician }\end{array}$ & $\begin{array}{l}350 \\
(53.8 \%)\end{array}$ & $\begin{array}{l}190 \\
(55.7 \%)\end{array}$ & 0.57 & - & $\begin{array}{l}252 \\
(53.6 \%)\end{array}$ & $\begin{array}{l}288 \\
(55.3 \%)\end{array}$ & 0.60 & \\
\hline $\begin{array}{l}\text { Referral } \\
\text { rights to sports } \\
\text { medicine }\end{array}$ & $\begin{array}{l}542 \\
(83.5 \%)\end{array}$ & $\begin{array}{l}248 \\
(72.7 \%)\end{array}$ & $<0.01$ & $\begin{array}{l}1.90 \\
{[1.39} \\
2.60]\end{array}$ & $\begin{array}{l}391 \\
(83.2 \%)\end{array}$ & $\begin{array}{l}399 \\
(76.7 \%)\end{array}$ & 0.01 & $\begin{array}{l}1.50 \\
{[1.09,} \\
2.06]\end{array}$ \\
\hline
\end{tabular}

specialist

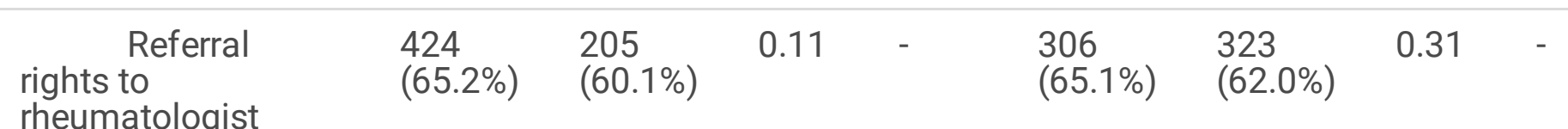

rheumatologist

Referral

rights to other

medical specialist

\begin{tabular}{l}
\multicolumn{1}{c}{ Expanded } \\
diagnostic imaging \\
rights \\
$\begin{array}{l}\text { Research ('strongly } \\
\text { agree') }\end{array}$
\end{tabular} understand

osteopathy

Help general

practitioners and

other health

professionals

understand

osteopathy

Orovide

scientific evidence $\stackrel{1}{(0.2 \%)}$

$\begin{array}{ll}551 & 271 \\ (84.8 \%) & (79.5 \%)\end{array}$

$0(0 \%)$
$0.46-0(0 \%)$

0.46

0.03

1.44

[1.02,
$1 \quad 0.35$

(0.2\%)

401

$(85.3 \%) \quad(80.8 \%)$

0.06 
0.98]

Irrelevant to
the development of osteopathy*

348

(56.4\%)
215

(67.2\%)
$<0.01$

0.63

$[0.47$, $0.84]$
261

(58.8\%)
302

(61.3\%)
$0.44-$

* 'strongly disagree'

Backward regression modelling of characteristics associated with sending patient referrals to a podiatrist is shown in Table 4. Osteopaths who reported sending referrals to podiatrists were more than twice as likely as those who did not send referral to be co-located with a podiatrist and more than ten times as likely to receive referrals from podiatrists. Sending referrals to podiatrists was also more likely to be associated with osteopaths treating postural disorders and using trigger point therapy and sports taping, compared with osteopaths who did not report sending referrals to podiatrists. 
Table 4

Adjusted odds ratios for significant practitioner and clinical management characteristics associated with Australian osteopaths sending referrals to podiatrists.

\begin{tabular}{|c|c|c|c|}
\hline & Odds ratio & $95 \% \mathrm{Cl}$ & p-value \\
\hline \multicolumn{4}{|l|}{ Practitioner characteristics ('female') } \\
\hline Gender & 0.38 & $0.24,0.60$ & $<0.01$ \\
\hline \multicolumn{4}{|l|}{ Practice characteristics ('yes') } \\
\hline More than one practice location & 0.49 & $0.31,0.77$ & 0.002 \\
\hline Co-located with a specialist & 0.07 & $0.02,0.25$ & $<0.01$ \\
\hline Co-located with a podiatrist & 2.87 & $1.36,6.03$ & 0.005 \\
\hline Co-located with an exercise physiologist & 2.88 & $1.88,7.01$ & $<0.01$ \\
\hline Send referrals to a physiotherapist & 3.39 & $2.09,5.49$ & $<0.01$ \\
\hline Send referrals to an exercise physiologist & 2.04 & $1.23,3.39$ & 0.006 \\
\hline Send referrals to a massage therapist & 1.77 & $1.13,2.75$ & 0.011 \\
\hline Send referrals to an acupuncturist & 1.78 & $1.14,2.79$ & 0.012 \\
\hline Receive referrals from a podiatrist & 10.82 & $6.66,17.58$ & $<0.01$ \\
\hline Receive referrals from an exercise physiologist & 0.46 & $0.25,0.85$ & 0.012 \\
\hline \multicolumn{4}{|l|}{ Practice characteristics ('often') } \\
\hline Use diagnostic imaging to assist with diagnosis & 9.87 & $1.64,59.13$ & 0.012 \\
\hline Use a screening questionnaire to assist with diagnosis & 1.68 & $1.08,2.61$ & 0.007 \\
\hline \multicolumn{4}{|l|}{ Clinical management characteristics ('often') } \\
\hline Treat postural disorders & 1.84 & $1.18,2.87$ & 0.007 \\
\hline Use joint manipulation & 1.68 & $1.08,2.61$ & 0.021 \\
\hline Use balanced ligamentous tension & 0.61 & $0.38,0.96$ & 0.031 \\
\hline Use of trigger point therapy & 2.99 & $1.77,5.06$ & $<0.01$ \\
\hline Frequency of use - sports taping & 1.25 & $1.00,1.59$ & 0.023 \\
\hline
\end{tabular}

Australian osteopaths who reported referring patients to podiatrists were $80 \%$ more likely to report receiving referrals from other allied health and complementary medicine professionals than osteopaths who did not report sending referrals to podiatrists (Table 5). Osteopaths who reported receiving referrals from podiatrists were nearly $70 \%$ more likely than those who did not receive referrals to have been 
involved in clinical supervision of osteopathy students in the 12 months before completion of the questionnaire.

\section{Table 5}

Adjusted odds ratios for significant practitioner and clinical management characteristics associated with Australian osteopaths receiving referrals from podiatrists.

\begin{tabular}{|llll|}
\hline & $\begin{array}{l}\text { Adjusted odds } \\
\text { ratio }\end{array}$ & $95 \%$ Cl & p-value \\
\hline Practitioner characteristics & & & \\
\hline Patient visits per week & 1.03 & $1.02,1.04$ & $<0.01$ \\
\hline Involved in clinical supervision ('yes') & 1.67 & $1.03,2.71$ & 0.037 \\
\hline Practice characteristics ('yes') & & & \\
\hline Co-located with a podiatrist & 2.02 & $1.20,3.41$ & 0.008 \\
\hline Send referrals to a podiatrist & 10.04 & $6.52,15.45$ & $<0.01$ \\
\hline Send referrals to a physiotherapist & 0.64 & $0.42,0.96$ & 0.033 \\
\hline Send referrals to an acupuncturist & 1.78 & $1.14,2.79$ & 0.012 \\
\hline $\begin{array}{l}\text { Receive referrals from a specialist medical } \\
\text { practitioner }\end{array}$ & 1.72 & $1.12,2.66$ & 0.013 \\
\hline Receive referrals from a physiotherapist & 1.83 & $1.17,2.87$ & 0.008 \\
\hline Receive referrals from a massage therapist & 2.12 & $1.35,3.34$ & 0.001 \\
\hline Receive referrals from a naturopath & 1.52 & $1.04,2.21$ & 0.029 \\
\hline Receive referrals from a nutritionist & 2.30 & $1.01,5.23$ & 0.046 \\
\hline Clinical management characteristics ('often') & & & \\
\hline Use TENS & 0.22 & $0.05,0.97$ & 0.046 \\
\hline
\end{tabular}

\section{Discussion}

The current study identified the demographic, practice and clinical management characteristics of Australian osteopaths who send referrals to, and receive referrals from, podiatrists. A significant finding was the number of osteopaths reporting referrals with podiatrists Approximately half of the sample of osteopaths reported receiving referrals from podiatrists, and approximately two-thirds reported sending referrals to podiatrists [5]. These results support the finding that a strong referral relationship exists between Australian osteopaths and podiatrists, and potentially reflect a shared understanding between osteopaths and podiatrists of the role that each profession plays in patient care. Evidence of Australian osteopaths engaging in multidisciplinary care is beginning to emerge in the literature, and the data from some studies indicate instances of multidisciplinary care involving podiatrists as part of the team [24-26]. 
These studies describe podiatrists positively contributing to the multidisciplinary care team for arthritic and ulcerative conditions; however, there is little literature describing this in the musculoskeletal care context.

Through secondary analysis of data from a national osteopathy PBRN, it appears that Australian osteopaths who report referral relationships with podiatrists are likely to engage in referrals with other allied health and complementary medicine professions. This finding is encouraging given the need for interprofessional patient care and has rarely been demonstrated in the literature describing the Australian osteopathy profession. The data presented here is also invaluable for informing health policy development around interprofessional care and in patient care for chronic conditions. There is also an opportunity to use the data in the current study to inform the pre-registration education of Australian osteopaths and podiatrists, particularly focusing on interprofessional care.

Regarding practitioner characteristics, the current study identified two significant variables. The first was that female osteopaths were $30 \%$ less likely to send referrals to podiatrists and $30 \%$ more likely to receive referrals from podiatrists compared to their male counterparts in the unadjusted modelling. The former finding was expressed in the adjusted model, where female Australian osteopaths were found to be $70 \%$ less likely to send referrals to podiatrists. The reason for this difference in referrals based on gender requires additional exploration. Those Australian osteopaths who reported receiving referrals from podiatrists were nearly $70 \%$ more likely to report having been involved in osteopathy clinical supervision in the previous 12 months compared with osteopaths who reported not receiving podiatry referrals. Again, the reason for this finding is not clear. However, it may be related to the aforementioned referral relationships and could prove promising in encouraging osteopathy students to engage in, and model, multidisciplinary patient care, particularly with podiatrists.

From a clinical management perspective, Australian osteopaths who send referrals to podiatrists were $80 \%$ more likely to report treating postural disorders. Although a direct relationship cannot be established from the current dataset, it would be reasonable to suggest that postural disorders may be a commonly referred condition for shared management of patients. Studies have described the relationship between the prescription of foot orthoses and changes in posture in both healthy $[27,28]$ and clinical populations [29]. Whether postural disorders are a key driver of referrals would be an interesting avenue for further research. In particular, it would be valuable to understand the clinical reasoning of osteopaths and podiatrists in the management of postural disorders. Additional research could also be directed towards understanding other conditions that could result in frequent referrals between the two professions.

The regression analysis undertaken indicates the payment processes that might be associated with osteopathy and podiatry co-management of patients. Australian osteopaths who send referrals to, or receive referrals from, podiatrists were over $30 \%$ more likely to use the Medicare EasyClaim service than osteopaths who do not send or receive such referrals. Both professions are eligible to treat patients under the CDM scheme, whereby patients with chronic diseases can access government funded care for up to five consultations with allied health professionals [13]. Medicare EasyClaim allows patients to claim the 
rebate at the time of the consultation. It may be that patients under this CDM scheme are utilising both osteopathy and podiatry services, and the increased use of Medicare EasyClaim reflects this. However, this assertion would require additional investigation.

The limitation of the current research is associated with the cross-sectional nature of the questionnaire. Questionnaires used with these study designs are potentially susceptible to social desirability and acquiescence biases [30], and the latter may have influenced responses to the send and receive referral items on the questionnaire. Further, it is not possible to comment on the frequency of referrals between the two professions given the dichotomous nature of the responses to these items.

\section{Conclusions}

This study offers an important empirical examination of the referral relationships between Australian osteopaths and podiatrists. Our secondary analysis of ORION PBRN data shows Australian osteopaths who report referral relationships with podiatrists are also more? likely to engage in referrals with other allied health and complementary medicine professions. The data presented here offers an invaluable resource for informing health policy development, and has the potential for use in the pre-registration education of Australian osteopaths and podiatrists. Further research is now required to develop a deeper understanding of the nature and frequency of the referrals between Australian osteopaths and podiatrists, including how these two professions work together through the Medicare CDM scheme for the benefit of patients.

\section{Declarations}

\section{Ethics approval and consent to participate}

Ethics approval for the current study was provided by the University of Technology Sydney (\#2014000759).

\section{Consent for publication}

Not applicable

\section{Availability of data and materials}

The datasets analysed during the current study are not publicly available as the authors do not have the authority to disseminate the data. The dataset is available through reasonable request at arccim@uts.edu.au.

\section{Competing interests}


The authors report no competing interests in relation to the manuscript.

\section{Funding}

The ORION project is funded by Osteopathy Australia. The funding source had no influence in the design of the study and collection, analysis, and interpretation of data and in writing the manuscript. The research reported in this paper is the sole responsibility of the authors and reflects the independent ideas and scholarship of the authors alone.

\section{Authors' contributions}

MF was involved in the conceptualisation, methodology, formal analysis, investigation, and writing the original draft and review and editing of the final draft.

BV was involved in the conceptualisation, methodology, formal analysis, investigation, and writing the original draft and review and editing of the final draft.

$A B$ was involved in writing the original draft and reviewing and editing the final draft.

SG was involved in writing the original draft and reviewing and editing the final draft.

KF was involved in writing the original draft and reviewing and editing the final draft.

GM was involved in writing the original draft and reviewing and editing the final draft.

\section{Acknowledgements}

The authors would like to acknowledge the support of Distinguished Professor Jon Adams, Dr Amie Steel and Dr Wenbo Peng as part of the ORION PBRN Scientific Committee.

\section{References}

1. NSW Health. Multidisciplinary Team Care. 2014.

https://www.health.nsw.gov.au/healthone/Pages/multidisciplinary-team-care.aspx. Accessed May 13th 2020.

2. Leach B, Morgan P, de Oliveira JS, Hull S, Østbye T, Everett C. Primary care multidisciplinary teams in practice: a qualitative study. BMC Family Pract. 2017; 18:115

3. Bonciani M, Barsanti S, Murante AM. Is the co-location of GPs in primary care centres associated with a higher patient satisfaction? Evidence from a population survey in Italy. BMC Health Serv Res. $2017 ; 17: 248$ 
4. Rumball-Smith J, Wodchis WP, Koné A, Kenealy T, Barnsley J, Ashton T. Under the same roof: colocation of practitioners within primary care is associated with specialized chronic care management. BMC Family Pract. 2014; 15:149

5. Adams J, Sibbritt D, Steel A, Peng W. A workforce survey of Australian osteopathy: analysis of a nationally-representative sample of osteopaths from the Osteopathy Research and Innovation Network (ORION) project. BMC Health Serv Res. 2018; 18:352. doi: 10.1186/s12913-018-3158-y.

6. Burke SR, Myers R, Zhang AL. A profile of osteopathic practice in Australia 2010-2011: a cross sectional survey. BMC Musculo Disorder. 2013; 14:227. doi: 10.1186/1471-2474-14-227.

7. Orrock P. Profile of members of the Australian Osteopathic Association: Part 2 - The patients. Int J Osteopath Med. 2009; 12:128-139. doi: 10.1016/j.ijosm.2009.06.001.

8. Podiatry Board of Australia. Accreditation. 2019. https://www.podiatryboard.gov.au/Accreditation.aspx. Accessed 22 April 2020.

9. Osteopathy Board of Australia. Accreditation. 2016. https://www.osteopathyboard.gov.au/Accreditation.aspx. Accessed 22 April 2020.

10. Podiatry Board of Australia. Registrant Data (Reporting period: 01 July 2019 to 30 September 2019). 2019. https://www.podiatryboard.gov.au/About/Statistics.aspx. Accessed February 13, 2020

11. Osteopathy Board of Australia. Registrant Data (Reporting period: 01 July 2019 to 30 September 2019). 2019. https://www.osteopathyboard.gov.au/About/Statistics.aspx. Accessed 13 February 2020.

12. Department of Health. Podiatry - Health workforce data. 2016. https://hwd.health.gov.au/webapi/customer/documents/factsheets/2016/Podiatry\%20\%202016.pdf. Accessed 13 May 2020.

13. Department of Health. Chronic Disease Management (formerly Enhanced Primary Care or EPC) - GP services. 2017. http://www.health.gov.au/internet/main/publishing.nsf/Content/mbsprimarycarechronicdiseasemanagement. Accessed 21 December 2107.

14. Menz HB. Utilisation of podiatry services in Australia under the Medicare Enhanced Primary Care program, 2004-2008. J Foot Ankle Res. 2009; 2:30

15. Department of Health. Medicare item reports. 2020. http://medicarestatistics.humanservices.gov.au/statistics/mbs_item.jsp. Accessed February 16 2020.

16. Pirotta M, Temple-Smith M. Practice-based research networks. Aust Fam Physician. 2017; 46:793795

17. Mold JW, Peterson KA. Primary care practice-based research networks: working at the interface between research and quality improvement. Ann Fam Med. 2005; 3:S12-S20

18. Adams J, Lauche R, Peng W, Steel A, Moore C, Amorin-Woods L, Sibbritt D. A workforce survey of Australian chiropractic: the profile and practice features of a nationally representative sample of 2,005 chiropractors. BMC Complement Altern Med. 2017; 17:14. doi: 10.1186/s12906-016-1542-x. 
19. Hickner J, Green LA. Practice-based research networks (PBRNs) in the United States: growing and still going after all these years. J Am Board Fam Med. 2015; 28:541-545. doi: 10.3122/jabfm.2015.05.150227.

20. Selby K, Cornuz J, Senn N. Establishment of a representative practice-based research network (PBRN) for the monitoring of primary care in Switzerland. J Am Board Fam Med. 2015; 28:673-675. doi: 10.3122/jabfm.2015.05.150110.

21. Gilbert GH, Williams OD, Rindal DB, Pihlstrom DJ, Benjamin PL, Wallace MC, Group DC. The creation and development of the dental practice-based research network. J Am Dent Assoc. 2008; 139:74-81

22. Steel A, Peng W, Sibbritt D, Adams J. Introducing national osteopathy practice-based research networks in Australia and New Zealand: an overview to inform future osteopathic research. Sci Rep. 2020; 10:846. doi: 10.1038/s41598-020-57918-7.

23. Paterson KL, Harrison C, Britt H, Hinman RS, Bennell KL. Management of foot/ankle osteoarthritis by Australian general practitioners: an analysis of national patient-encounter records. Osteoarthritis Cartilage. 2018; 26:888-894

24. Ndosi M, Ferguson R, Backhouse MR, Bearne L, Ainsworth P, Roach A, Dennison E, Cherry L. National variation in the composition of rheumatology multidisciplinary teams: a cross-sectional study. Rheumatol Int. 2017; 37:1453-1459

25. Cassidy S, Coda A, West K, Hendry G, Grech D, Jones J, Hawke F, Singh-Grewal D. Confidence amongst multidisciplinary professionals in managing paediatric rheumatic disease in Australia. Arthritis. 2018; 2018

26. Butters T. Interdisciplinary chronic-wound care services involving podiatry-a strengthened model of care? Wound Practice \& Research. 2011; 19:229

27. Olmsted LC, Hertel J. Influence of foot type and orthotics on static and dynamic postural control. J Sport Rehabil. 2004; 13:54-66

28. Wilson ML, Rome K, Hodgson D, Ball P. Effect of textured foot orthotics on static and dynamic postural stability in middle-aged females. Gait Posture. 2008; 27:36-42

29. Simonsen MB, Oliveira ASC, Næsborg-Andersen K, Kowalski MR, Hørslev-Petersen K, Andersen MS. Patient-specific foot orthotics improves postural control of rheumatoid arthritis patients: a pilot study. Footwear Sci. 2017; 9:S17-S18

30. Lavrakas P: Encyclopedia of Survey Research Methods. California, USA: Sage Publications; 2008. 